Leslie, K.; Myles, P.; Devereaux, P.J.; Forbes, A.; Rao-Melancini, P.; Williamson, E.; Xu, S.C.; Foex, P.; Pogue, J.; Arrieta, M.; Bryson, G.L.; Paul, J.; Paech, M.J.; Merchant, R.N.; Choi, P.T.; Badner, N.; Peyton, P.; Sear, J.W.; Yang, H. (2013). Nitrous Oxide and Serious Morbidity and Mortality in the POISE Trial. ANESTHESIA AND ANALGESIA, 116(5), 1034-1040.

Copyright (c) 2000-2014 Ovid Technologies, Inc.

This is a non-final version of an article published in final form in Anesthesia and Analgesia. The definitive published version (see citation above) is located on the article abstract page of the publisher, Lippincott Williams \& Wilkins.

This version was made available in the UWA Research Repository on 1 May 2014 in compliance with the publisher's policies on archiving in institutional repositories.

Use of the article is subject to copyright law. 


\section{Nitrous Oxide and Serious Morbidity and Mortality in the POISE Trial}

\section{Kate Leslie, MBBS, MD, MEpi, FANZCA}

Title: Professor

Affiliation: Department of Anaesthesia and Pain Management, Royal Melbourne Hospital, Melbourne, Australia; Department of Pharmacology, University of

Melbourne, Melbourne, Australia

Email: kate.leslie@mh.org.au

Role: This author helped design the study, conduct the study, and write the manuscript Conflicts: Kate Leslie reported no conflicts of interest

Attestation: Kate Leslie reviewed the analysis of the data, approved the final manuscript, and is the author responsible for archiving the study files

\section{Paul Myles, MBBS, MD, MPH, FANZCA, FCARSCI, FRCA}

Title: Professor

Affiliation: Department of Anaesthesia and Perioperative Medicine, Alfred Hospital, Melbourne, Australia; Academic Board of Anaesthesia and Perioperative Medicine, Monash University, Melbourne, Australia; National Health and Medical Research Council Practitioner Fellow

Email: p.myles@alfred.org.au

Role: This author helped design the study, conduct the study, and write the manuscript Conflicts: Paul Myles reported no conflicts of interest

Attestation: Paul Myles reviewed the analysis of the data and approved the final manuscript

\section{Philip J Devereaux, MD, PhD}

Title: $\mathrm{Dr}$

Affiliation: Departments of Medicine and Clinical Epidemiology and Biostatistics, McMaster University, Hamilton, Canada

Email:philipj@mcmaster.ca

Role: This author helped design the study, conduct the study, and write the manuscript Conflicts: Philip J Devereaux reported no conflicts of interest

Attestation: Philip J Devereaux has seen the original study data, reviewed the analysis of the data, and approved the final manuscript

\section{Andrew Forbes, MSc, PhD}

Title: Professor

Affiliation: Department of Epidemiology and Preventive Medicine, Monash University, Melbourne, Australia

Email: andrew.forbes@monash.edu

Role: This author helped analyze the data and write the manuscript Conflicts: Andrew Forbes reported no conflicts of interest

Attestation: Andrew Forbes has seen the original study data, reviewed the analysis of the data, and approved the final manuscript

\section{Purnima Rao-Melancini, MSc}


Title: Ms

Affiliation: Population Health Research Institute, McMaster University, Hamilton, Canada

Email: Purnima.RaoMelacini@phri.ca

Role: This author helped analyze the data and write the manuscript

Conflicts: Purnima Rao-Melancini reported no conflicts of interest

Attestation: Purnima Rao-Melancini has seen the original study data, reviewed the analysis of the data, and approved the final manuscript

\section{Elizabeth Williamson, PhD}

Title: $\mathrm{Dr}$

Affiliation: Department of Epidemiology and Preventive Medicine, Monash University, Melbourne, Australia; School of Population Health, University of Melbourne,

Melbourne, Australia

Email: ewi@unimelb.edu.au

Role: This author helped analyze the data and write the manuscript

Conflicts: Elizabeth Williamson reported no conflicts of interest

Attestation: Elizabeth Williamson has seen the original study data, reviewed the analysis of the data, and approved the final manuscript

\section{Shouchun $\mathrm{Xu}, \mathrm{MD}$}

Title: $\mathrm{Dr}$

Affiliation: Fu Wai Cardiovascular Hospital, CAMS, Peoples Republic of China

Email: scxhypt@yahoo.com.cn

Role: This author helped conduct the study and write the manuscript

Conflicts: Shouchun Xu reported no conflicts of interest

Attestation: Shouchun Xu approved the final manuscript

\section{Pierre Foex, MD, DPhil, FRCA, FANZCA, FCA(SA), FMedSci}

Title: Professor

Affiliation: Nuffield Division of Anaesthetics, Oxford University, Oxford, United Kingdom

Email: pierre.foex@nda.ox.ac.uk

Role: This author helped conduct the study and write the manuscript

Conflicts: Pierre Foex reported no conflicts of interest

Attestation: Pierre Foex approved the final manuscript

\section{Janice Pogue, MSc}

Title: $\mathrm{Dr}$

Affiliation: Population Health Research Institute, McMaster University, Hamilton, Canada

Email: janice@phri.ca

Role: This author helped analyze the data and write the manuscript

Conflicts: Janice Pogue reported no conflicts of interest

Attestation: Janice Pogue has seen the original study data, reviewed the analysis of the data, and approved the final manuscript 
10. Maribel Arrieta, MD

Title: $\mathrm{Dr}$

Affiliation: Nueva Granada Military University, Bogota, Colombia; Department of Anaesthesia, Resuscitation and Pain Management, Central Military Hospital, Bogota, Colombia

Email: maribel.ao@gmail.com

Role: This author helped conduct the study and write the manuscript Conflicts: Maribel Arrieta reported no conflicts of interest

Attestation: Maribel Arrieta approved the final manuscript

\section{Gregory L. Bryson, MD, FRCPC, MSc}

Title: $\mathrm{Dr}$

Affiliation: Department of Anesthesiology, The Ottawa Hospital, Ottawa, Canada Email: glbryson@ottawahospital.on.ca

Role: This author helped conduct the study and write the manuscript Conflicts: Gregory L. Bryson reported no conflicts of interest Attestation: Gregory L. Bryson approved the final manuscript

\section{James Paul, MSc, MD, FRCPC}

Title: $\mathrm{Dr}$

Affiliation: Department of Anesthesia, McMaster University, Hamilton Health Sciences, Hamilton, Canada

Email: james_paul@sympatico.ca

Role: This author helped conduct the study and write the manuscript Conflicts: James Paul reported no conflicts of interest

Attestation: James Paul approved the final manuscript

\section{Michael J. Paech, MBBS, DM, DRCOG, FRCA, FANZCA, FFPMANZCA, FRANZCOG}

(Hon)

Title: Professor

Affiliation: School of Medicine and Pharmacology, University of Western Australia, Perth, Australia; Department of Anaesthesia and Pain Medicine, King Edward Memorial Hospital for Women, Perth, Australia

Email: Michael.Paech@health.wa.gov.au

Role: This author helped conduct the study and write the manuscript Conflicts: Michael J. Paech reported no conflicts of interest

Attestation: Michael J. Paech approved the final manuscript

\section{Richard N. Merchant, MD, FRCPC}

Title: $\mathrm{Dr}$

Affiliation: Department of Anesthesia and Perioperative Medicine, Royal Columbian Hospital, New Westminster, Canada

Email: richard.merchant@ubc.ca

Role: This author helped conduct the study and write the manuscript

Conflicts: Richard N. Merchant reported no conflicts of interest

Attestation: Richard N. Merchant approved the final manuscript 


\section{Peter T. Choi, MD, MSc, FRCPC}

Title: $\mathrm{Dr}$

Affiliation: Department of Anesthesiology, The University of British Columbia, Vancouver, Canada

Email: Peter.Choi2@vch.ca

Role: This author helped conduct the study and write the manuscript

Conflicts: Peter T. Choi reported no conflicts of interest

Attestation: Peter T. Choi approved the final manuscript

\section{Neal Badner , MD, FRCPC}

Title: $\mathrm{Dr}$

Affiliation: Department of Anesthesia; Perioperative Medicine, University of Western Ontario

Email: Neal.Badner@lhsc.on.ca

Role: This author helped conduct the study and write the manuscript

Conflicts: Neal Badner reported no conflicts of interest

Attestation: Neal Badner approved the final manuscript

\section{Philip Peyton, MBBS, MD, FANZCA}

Title: Associate Professor

Affiliation: Department of Anaesthesia, Austin Hospital, Melbourne, Australia;

Department of Surgery, Austin Hospital and University of Melbourne, Melbourne, Australia

Email: phil.peyton@austin.org.au

Role: This author helped conduct the study and write the manuscript

Conflicts: Philip Peyton reported no conflicts of interest

Attestation: Philip Peyton approved the final manuscript

\section{John W Sear, MA, BSc, MBBS, PhD, FFARCS, FANZCA}

Title: Professor

Affiliation: Nuffield Department of Anaesthetics, Oxford University, Oxford, United Kingdom

Email: john.sear@nda.ox.ac.uk

Role: This author helped conduct the study and write the manuscript

Conflicts: John W Sear reported no conflicts of interest

Attestation: John W Sear approved the final manuscript

\section{Homer Yang, MD, CCFP, FRCPC, CCPE}

Title: $\mathrm{Dr}$

Affiliation: Department of Anesthesia, University of Ottawa, Ottawa, Canada

Email: hyang@ottawahospital.on.ca

Role: This author helped conduct the study and write the manuscript

Conflicts: Homer Yang reported no conflicts of interest

Attestation: Homer Yang approved the final manuscript

Institution: Department of Anaesthesia and Pain Management, Royal Melbourne Hospital, 
Melbourne, Australia; Department of Pharmacology, University of Melbourne, Melbourne, Australia; Department of Anaesthesia and Perioperative Medicine, Alfred Hospital, Melbourne, Australia; Academic Board of Anaesthesia and Perioperative Medicine, Monash University, Melbourne, Australia; National Health and Medical Research Council, Canberra, Australia; Departments of Medicine and Clinical Epidemiology and Biostatistics, McMaster University, Hamilton, Canada; Department of Epidemiology and Preventive Medicine, Monash University, Melbourne, Australia; Population Health Research Institute, McMaster University, Hamilton, Canada; School of Population Health, University of Melbourne, Melbourne, Australia; Fu Wai Cardiovascular Hospital, CAMS, People's Republic of China; Nuffield Division of Anaesthetics, Oxford University, Oxford, United Kingdom; Nueva Granada Military University, Bogota, Colombia; Department of Anaesthesia, Resuscitation and Pain Management, Central Military Hospital, Bogota, Colombia; Department of Anesthesiology, The Ottawa Hospital, Ottawa, Canada; Department of Anesthesia, McMaster University, Hamilton Health Sciences, Hamilton, Canada; School of Medicine and Pharmacology, University of Western Australia, Perth, Australia; Department of Anaesthesia and Pain Medicine, King Edward Memorial Hospital for Women, Perth, Australia; Department of Anesthesia and Perioperative Medicine, Royal Columbian Hospital, New Westminster, Canada; Department of Anesthesiology, The University of British Columbia, Vancouver, Canada; Department of Anesthesia and Perioperative Medicine, University of Western Ontario; Department of Anaesthesia, Austin Hospital, Melbourne, Australia; Department of Surgery, Austin Hospital and University of Melbourne, Melbourne, Australia; Nuffield Department of Anaesthetics, Oxford University, Oxford, United Kingdom; Department of Anesthesia, University of Ottawa, Ottawa, Canada

Short Title: Nitrous Oxide and POISE

Funding: Canadian Institutes of Health Research; Australian National Health and Medical Research Council Instituto de Salud Carlos

\section{Corresponding Author:}

Kate Leslie, MBBS, MD, MEpi, FANZCA

Department of Anaesthesia and Pain Management, Royal Melbourne Hospital, Melbourne, Australia; Department of Pharmacology, University of Melbourne, Melbourne, Australia Department of Anaesthesia and Pain Management, Royal Melbourne Hospital, Parkville, VIC, 3050, Australia

Phone: +61-3-93427540

FAX: +61-3-93428623

Email: kate.leslie@mh.org.au

Information for LWW regarding depositing manuscript into PubMed Central: This paper does not need to be deposited in PubMed Central.

For Editorial Office: This report was previously presented, in part, at the ANZCA ASM 2009 Cairns Australia 
This report describes human research. IRB contact information: Dr Angela Watt Director Research Governance and Ethics, Melbourne Health, Royal Melbourne Hospital, Parkville, VIC, 3050, Australia. Telephone: +61-3-9342 7550 Fax: +61-39342 8548 Email: angela.watt@mh.org.au

This study was conducted with written informed consent from the study subjects.

This report describes an observational clinical study.

This report describes cohort observational clinical study. The author states that the report includes every item in the STROBE checklist for cohort observational clinical studies.

This manuscript was screened for plagiarism using Doc Cop.

Link to Title Page: http://www.aaauthor.org/pages/4098-2011-Nov-30 


\section{Abstract}

Background: The aim of this post-hoc sub-analysis of the POISE trial was to determine whether nitrous oxide was associated with the primary composite outcome of cardiovascular death, non-fatal myocardial infarction and non-fatal cardiac arrest within 30 days of randomization.

Methods: The POISE Trial of perioperative beta-blockade was undertaken in 8,351 patients. Nitrous oxide anesthesia was defined as co-administration of nitrous oxide in patients receiving general anesthesia, with or without additional neuraxial blockade or peripheral nerve blockade. Logistic regression, with inverse probability weighting using estimated propensity scores, was used to determine the association of nitrous oxide with the primary outcome, myocardial infarction, stroke, death and clinically significant hypotension.

Results: Nitrous oxide was administered to 1,489 (29\%) of the 5,133 patients included in this analysis. Nitrous oxide had no significant effect on the risk of the primary outcome (112 [7.5\%] vs. 248 [6.9\%]; OR, 1.08; 95\% Cl, 0.82-1.44; $99 \% \mathrm{Cl}, 0.75-1.57 ; \mathrm{P}=0.58)$, myocardial infarction (89 [6.0] vs. 204 [5.6]; OR, 0.99; 95\% Cl, 0.75-1.31; 99\% Cl, 0.69-1.42; P = 0.94), stroke (6 [0.4\%] vs. 28 [0.8\%]; OR, 0.85; 95\% Cl, 0.26-2.82; 99\% Cl, 0.17-4.11; $\mathrm{P}=0.79$ ), death (40 [2.7\%] vs. 100 [2.8\%]; OR, 1.04; 95\% Cl, 0.6-1.81; 99\% $\mathrm{Cl}, 0.51-2.15 ; \mathrm{P}=0.88$ ) or clinically significant hypotension (219 [14.7\%] vs. 544 [15.0\%]; OR, 0.92; 95\% Cl, 0.74-1.15; $99 \% \mathrm{Cl}, 0.70-1.23 ; \mathrm{P}=0.48)$.

Conclusions: In this post-hoc sub-analysis, nitrous oxide was not associated with an increased risk of adverse outcomes in POISE Trial patients. This analysis was limited by the 
observational nature of the data and lack of information on the concentration and duration of nitrous oxide administration. Further randomized controlled trial evidence is required. 


\section{Introduction}

Nitrous oxide is commonly used during non-cardiac surgery and, in view of the large number of patients exposed worldwide every year, good evidence for its beneficial or harmful effects is desirable. However, the available large randomized trial ${ }^{1}$ and observational studies $^{2-4}$ report conflicting results which limit reliable conclusions about the value of nitrous oxide.

Nitrous oxide may reduce potent hypnotic and opioid requirements intraoperatively and improve acute and chronic pain outcomes postoperatively. ${ }^{5}$ However, the effect of nitrous oxide on perioperative cardiac, pulmonary, and thrombotic outcomes is unclear. ${ }^{1-4}$ Nitrous oxide use may increase the risk of myocardial infarction (MI) via increased plasma homocysteine concentrations and endothelial dysfunction after surgery. ${ }^{3}$ Investigating the effectiveness of anesthetic management using randomized trials is challenging ${ }^{6,7}$ and so analysis of large prospectively collected datasets is warranted. ${ }^{4}$

The POISE Trial randomized 8,351 patients with or at risk of ischemic heart disease having non-cardiac surgery to 30 days of extended-release metoprolol succinate or placebo. ${ }^{8}$ Metoprolol was associated with a decreased risk of non-fatal MI (4.2\% versus 5.7\%; $p=$ $0.002)$ but an increased risk of stroke $(1.0 \%$ versus $0.5 \% ; p=0.005)$ and death $(3.1 \%$ versus $2.1 \% ; p=0.03$ ) compared with placebo. POISE required complete prospective collection of many relevant indices throughout the 30-day study period. With the significant adverse event rates and the substantial use of nitrous oxide in the trial, this dataset provides an opportunity to further explore the effects of nitrous oxide on major outcomes, even though this was not the original aim of POISE. The primary aim of this post-hoc sub-analysis, 
therefore, was to determine the effects of nitrous oxide on the incidence of a composite outcome of cardiovascular death, non-fatal MI and non-fatal cardiac arrest occurring within 30 days after randomization in the POISE Trial patients. 


\section{Materials and Methods}

The POISE Trial protocol has been described in detail elsewhere and was registered with ClinicalTrials.gov (NCT00182039). ${ }^{8,9}$ In summary, patients were eligible for this multi-center, blinded, randomized controlled trial, if they were aged $\geq 45$ years, were undergoing noncardiac surgery with an expected hospital length of stay $\geq 24 \mathrm{~h}$, and fulfilled at least one of the following criteria: history of coronary artery disease, peripheral vascular disease, stroke, hospitalization for congestive heart failure, undergoing major vascular surgery or any three of seven criteria (intrathoracic or intraperitoneal surgery, history of congestive heart failure, transient ischemic attack, diabetes, serum creatinine $>175 \mu \mathrm{mol} / \mathrm{L}$, age $>70$ years or undergoing emergency surgery). Patients were excluded if they met the following criteria: heart rate $<50$ beats per minute, second or third degree heart block, asthma, already receiving a beta-blocker, coronary artery bypass surgery within five years and no cardiac ischemia since, low-risk surgical procedures, on verapamil or previous randomization into the trial. All centers obtained institutional review board approval and all patients provided informed consent for the original trial. Patients were only recruited once and data relate to the index surgery and not any other surgery. Patients who were related to another participant were not excluded. Institutional review board pendency was not maintained, and approval to add investigators who assisted with these analyses was not sought (these investigators did not have access to identifiable data nor individual case report forms).

A total of 8,351 patients from 190 hospitals in 23 countries were randomly assigned to extended-release metoprolol succinate or placebo, starting 2-4 $\mathrm{h}$ before surgery and continuing for 30 days. Study medication was only administered if the heart rate was $\geq 50$ beats per minute and the systolic blood pressure was $\geq 100 \mathrm{~mm} \mathrm{Hg}$. Patients were 
monitored with cardiac biomarker assays and electrocardiographs during the 30-day followup period and cardiovascular outcomes were adjudicated centrally by a blinded committee. The dosage and monitoring regimens were described in detail previously. ${ }^{8,9}$

The primary outcome was a composite of cardiovascular death, non-fatal MI and non-fatal cardiac arrest occurring within 30 days of randomization. Secondary outcomes included MI and stroke. Clinically significant hypotension was defined as a systolic blood pressure of $<90$ $\mathrm{mm} \mathrm{Hg}$ requiring fluid resuscitation, an inotropic agent or study drug discontinuation or intraaortic balloon pump. ${ }^{9}$ Testing the effect of nitrous oxide on these outcomes was not the primary purpose for which the POISE Trial was designed.

Nitrous oxide anesthesia was defined as co-administration of nitrous oxide in patients receiving general anesthesia, with or without additional neuraxial blockade or nerve blockade.

\section{Data analysis}

Baseline characteristics were summarized as number (\%) for categorical variables and mean (standard deviation) for continuous variables, and were compared between intervention groups using chi-squared tests and analysis of variance, respectively.

Use of nitrous oxide was left to the discretion of the attending anesthesiologists; that is, such use was not randomly assigned. Patient characteristics were therefore imbalanced between intervention groups, and so we used a propensity score technique to account for potential confounding. The propensity score is the probability of receiving the intervention, modelled as a function of observed variables, and can be used in various ways to adjust for confounding due to observed characteristics. ${ }^{10,11}$ We adopted an inverse probability 
weighted approach, which uses the propensity score to create a 'pseudo-population' in which all measured characteristics are balanced between the intervention groups, hence removing any confounding by these characteristics (but not necessarily by unmeasured or unknown confounders). ${ }^{12}$ This is akin to the balance that is expected to be observed amongst measured variables in a randomized trial; however, it is not akin to the balance one would expect with unknown prognostic variables in a large randomized trial. A key assumption in the use of such methods is that every patient must have a non-zero probability of receiving each studied intervention. For nitrous oxide, patients not receiving a general anesthetic were excluded.

The propensity score for nitrous oxide use was estimated using a logistic regression model in which the outcome was the intervention group. An iterative procedure was used to select independent variables to include in the model, initially including main effects for all characteristics listed in Tables 1 and 2, adding interaction terms until no further imbalance could be removed. All analyses were repeated using a more comprehensive propensity score model including all region-by-covariate interactions, in order to investigate and account for geographical differences in intervention allocation.

We then imposed the 'common support' condition ${ }^{11}$ in which we excluded any patients in the intervention group who had an estimated propensity score higher than that of any patient in the no-intervention group, and any patients in the no-intervention group with a propensity score lower than that of any patient receiving the intervention. This removed subjects for whom no comparable subject existed in the other intervention group, since the effect of the intervention cannot be estimated for such patients. 
Each subject was inversely weighted by the probability of that subject receiving the intervention that they did indeed receive (calculated using the propensity score). Within the weighted sample (the 'pseudo-population'), measured patient prognostic characteristics should be balanced between the intervention groups. This was assessed using standardized differences (the difference in the percentage [or mean] of each characteristic between intervention groups, divided by an estimate of the standard deviation and expressed as a percentage $)^{13}$ calculated both for the original sample and the weighted sample. It has been suggested that a standardized difference of $10 \%$ or greater represents a meaningful imbalance. $^{14}$

Odds ratios (OR) and $95 \%$ confidence intervals ( $\mathrm{Cls}$ ) for the effect of nitrous oxide on the primary composite outcome, $\mathrm{MI}$ and stroke were estimated using logistic regression models for each outcome including the intervention group as the sole independent variable by applying a weighted analysis as described above. Characteristics that remained imbalanced in the weighted sample were additionally included as independent variables in the logistic regression model to remove any residual confounding by these variables.

To assess between-region variability in effect, an interaction between intervention and geographical region was additionally included in the weighted logistic regression model. Estimated associations with the outcome primary for nitrous oxide by region were also calculated.

To assess the sensitivity of results to a few individuals with large weights, we excluded the $1 \%$ of subjects with the largest weights and repeated all the analyses. Subjects with missing data for surgery type or baseline heart rate or systolic blood pressure were excluded from all analyses. Analyses were conducted using Stata version 11.1. A P $<0.05$ was considered statistically significant. 


\section{Results}

For nitrous oxide, 5,104 patients remained after exclusions were made for patients who did not receive general anaesthesia $(n=3,139)$, for those with missing data $(n=30)$, and for the common support condition $(n=78)$.

Patients receiving nitrous oxide were more likely to have ischemic heart disease and were less likely to be having intra-abdominal or emergency surgery than those not receiving nitrous oxide (Table 1). There was also significant regional variation in the administration of nitrous oxide, ranging from $5 \%$ in Central/South America to $80 \%$ in India. Imbalances between the intervention groups in the initial sample were reduced to minimal levels by the propensity score weighting (Tables 1 ). In particular, the groups were well balanced for the randomized beta-blocker treatment.

There was no evidence of an association between nitrous oxide and the risk of the primary outcome (OR, 1.08; 95\% Cl, 0.82-1.44; $99 \% \mathrm{Cl}, 0.75-1.57 ; \mathrm{P}=0.58)$, MI (OR, 0.99; 95\% Cl, $0.75-1.31 ; 99 \% \mathrm{Cl}, 0.69-1.42 ; \mathrm{P}=0.94)$ or stroke $(\mathrm{OR}, 0.85 ; 95 \% \mathrm{Cl}, 0.26-2.82 ; 99 \% \mathrm{Cl}, 0.17-$ $4.11 ; \mathrm{P}=0.79)$, death $(\mathrm{OR}, 1.04 ; 95 \% \mathrm{Cl}, 0.6-1.81 ; 99 \% \mathrm{Cl}, 0.51-2.15 ; \mathrm{P}=0.88)$ or clinically significant hypotension (OR, 0.92; $95 \% \mathrm{Cl}, 0.74-1.15 ; 99 \% \mathrm{Cl}, 0.70-1.23 ; \mathrm{P}=0.48$ ) (Table 2). Trimming the weights modified the odds ratios slightly $(1.14,1.13,0.63,0.88$ and 0.94 , respectively), however all P-values remained above 0.27 thereby retaining the conclusions from the untrimmed data. There was no evidence to support an effect of nitrous oxide on the primary outcome based on region (Table 3). 


\section{Discussion}

We found no evidence that nitrous oxide had an effect on the primary outcome (cardiovascular death, non-fatal MI and non-fatal cardiac arrest), MI, stroke or clinically significant hypotension in the POISE Trial patients. We did however find marked geographical differences in the rates of nitrous oxide administration, suggesting differences in regional preferences and ongoing uncertainty of the benefits and risks of nitrous oxide. Our results should, however, be considered in the light of the study's limitations, as outlined below.

This result is possibly due to a lack of power, but nevertheless is consistent with previous literature on this subject, ${ }^{1,3,4}$ despite the plausible pathophysiologic rationale ${ }^{3}$ and clinical trial support ${ }^{15}$ for increased myocardial ischemia in nitrous oxide patients. The trend towards an increased rate of death and MI at 30 days was not statistically significant in the ENIGMA-1 Trial, ${ }^{1}$ although a long-term follow-up revealed a marginal increase in the risk of MI. ${ }^{3}$ In contrast, Shiba et al. ${ }^{4}$ reported no effect on 30-day mortality and a decrease in hospital morbidity in nitrous oxide patients when analyzing a large administrative dataset. We thus have uncertainty regarding this important clinical issue for anesthesiologists. Consequently, we are conducting a randomized trial of nitrous oxide-based versus nitrous oxide-free anesthesia in 7,000 noncardiac surgery patients who have or are at risk of ischemic heart disease (the ENIGMA-II Trial; www.enigma2.org.au) ${ }^{6}$ The contrasting patterns of use around the world reported in the current study illustrate the uncertainty about the risk-benefit ratio for nitrous oxide and further support the need for compelling 
evidence. In the meantime, those anesthesiologists who omit nitrous oxide from their general anesthesia plan in high-risk patients should not do so on the basis that risk of important morbidity will definitely be reduced, because these results and others are not definitive.

The current analyses have several limitations. Firstly, the POISE Trial was not designed with the primary purpose of testing the effects of nitrous oxide on the primary or secondary outcomes. The patients in the POISE Trial were not randomized to nitrous oxide, and the use of this drug was at the discretion of the attending anesthesiologist. Although we included a propensity analysis in order to account for the decision to use nitrous oxide, we were limited by the data available. In addition, the original protocol was not developed with this analysis in mind. There are likely to be unmeasured and unknown factors that influenced the choice of nitrous oxide that may provide an alternative explanation for our findings. Interest in and experience with nitrous oxide use varies amongst anesthesiologists and there are certainly institutional and regional differences. Whilst nitrous oxide use is not commonly discussed with patients, surgeons may be concerned about the effects of nitrous oxide during bowel or middle ear surgery. ${ }^{16}$ In the present study, the only data point that captured these factors was the regional variations in the use of these techniques, and we included these in our propensity score.

Secondly, we did not record the details of the nitrous oxide use when they were administered (i.e. timing of initiation, inspired nitrous oxide concentration and duration of administration). Randomized trials on this intervention need to control or record these factors. The dose of nitrous oxide determines extent of hyperhomocysteinemia and its possible consequences. ${ }^{17}$ Finally, we did not collect data on other aspects of anesthesia 
care, such as the use of volatile-based or propofol-based maintenance of anesthesia, opioid use or postoperative care; on the actual operation undertaken or on the success of surgery (particularly in relation to vascular graft patency ). Nitrous oxide was the only component of the general anesthetic technique that was recorded.

In conclusion, we found no evidence that nitrous oxide was associated with any of these outcomes. These results have important implications for future research. 


\section{References}

1. Myles P, Leslie K, Chan M, Forbes A, Paech M, Peyton P, Silbert B, Pascoe E. Avoidance of nitrous oxide for patients undergoing major surgery: A randomized controlled trial. Anesthesiology 2007; 107: 221-31

2. Leslie K, Myles P, Forbes A, Chan M. The effect of bispectral index monitoring on long-term survival in the B-Aware trial. Anesth Analg 2010; 110: 816-22

3. Leslie K, Myles P, Chan M, Forbes A, Paech M, Peyton P, Silbert B, Williamson E. Nitrous oxide and long-term morbidity and mortality in the ENIGMA trial. Anesth Analg 2011; 112: 387-93

4. Shiba S, You J, Saager L, Sessler D, Turan A. Nitrous oxide and postoperative outcomes after non-cardiac surgery, American Society of Anesthesiologists Annual Meeting. San Diego, 2010

5. Stiglitz D, Amaratunge L, Konstantatos A, Lindholm D. Intraoperative nitrous oxide as a preventive analgesic. Anaesth Intensive Care 2010; 38: 890-3

6. Myles P, Leslie K, Peyton P, Paech M, Forbes A, Chan M, Sessler D, Devereaux P, Silbert B, Jamrozik K, Beattie S, Badner N, Tomlinson J, Wallace S. Nitrous oxide and perioperative cardiac morbidity (ENIGMA-II) Trial: Rationale and design. Am Heart J 2009; 157-62: 488-94

7. McPeek B. Inference, generalizability and a major change in anesthetic practice. Anesthesiology 1987; 66: 723-4

8. POISE Study Group. Effects of extended-release metoprolol succinate in patients undergoing non-cardiac surgery (POISE trial): a randomised controlled trial. Lancet 2008; 371: 1839-47 
9. POISE Trial Investigators. Rationale, design, and organization of the PeriOperative ISchemic Evaluation (POISE) Trial: A randomized controlled trial of metoprolol versus placebo in patients undergoing noncardiac surgery. Am J Heart 2006; 152: 223-230

10. Rosenbaum P, Rubin D. The central role of the propensity score in observational studies for causal effects. Biometrika 1983; 70: 41-55

11. Williamson E, Morley R, Lucas A, Carpenter J. Propensity scores: From naïve enthusiasm to intuitive understanding. Statistical Methods in Medical Research 2012; 21: 273-93

12. Hernan M, Robins J. Estimating causal effects from epidemiological data. Epidemiol Community Health 2006; 60: 578-86

13. Austin $\mathrm{P}$, Grootendorst $\mathrm{P}$, Anderson $\mathrm{G}$. A comparison of the ability of different propensity score models to balance measured variables between treated and untreated subjects: a Monte Carlo study. Statistics in Medicine 2007; 26: 734-53

14. Sharon-Lise T, Normand S-L T, Landruma M, Guadagnolia E, Ayaniana J, Ryand T, Clearya P, McNeil B. Validating recommendations for coronary angiography following acute myocardial infarction in the elderly: A matched analysis using propensity scores. Journal of Clinical Epidemiology 2001; 54 387-98

15. Badner N, Beattie W, Freeman D, Spence J. Nitrous oxide-induced increased homocysteine concentrations are associated with increased postoperative myocardial ischemia in patients undergoing carotid endarterectomy. Anesth Analg 2000; 91: 1073-9

16. Myles $\mathrm{P}$, Leslie $\mathrm{K}$, Silbert $\mathrm{B}$, Paech $\mathrm{M}$, Peyton P. A review of the risks and benefits of nitrous oxide in current anaesthetic practice. Anaesth Intensive Care 2004; 32: 165- 
17. Myles $\mathrm{P}$, Chan $\mathrm{M}$, Leslie $\mathrm{K}$, Peyton $\mathrm{P}$, Paech $\mathrm{M}$, Forbes $\mathrm{A}$. Effect of nitrous oxide on plasma homocysteine and folate in patients undergoing major surgery. Br J Anaesth 2008; 100: 780-6 
Table 1 Nitrous oxide baseline characteristics $(n=5,104)$

\begin{tabular}{|c|c|c|c|c|c|c|c|c|}
\hline & \multicolumn{4}{|c|}{ Unweighted - \% (N) } & \multicolumn{4}{|c|}{ Propensity score weighted - \% } \\
\hline & $\begin{array}{c}\text { No nitrous } \\
\text { oxide } \\
(n=3,616)\end{array}$ & $\begin{array}{l}\text { Nitrous } \\
\text { oxide } \\
(n=1,488)\end{array}$ & & & $\begin{array}{c}\text { No nitrous } \\
\text { oxide } \\
(n=3,616)\end{array}$ & $\begin{array}{c}\text { Nitrous } \\
\text { oxide } \\
(n=1,488)\end{array}$ & & \\
\hline Characteristic & $\%(N) *$ & $\%(\mathrm{~N})^{*}$ & $\begin{array}{c}\text { StDiff } \\
(\%)\end{array}$ & $\begin{array}{c}\mathrm{P} \\
\text { value }^{* *}\end{array}$ & $\%(N) *$ & $\%(\mathrm{~N})^{*}$ & $\begin{array}{c}\text { StDiff } \\
(\%)\end{array}$ & $\begin{array}{c}\mathrm{P} \\
\text { value }^{* *}\end{array}$ \\
\hline Age (years) & $69.7(10)$ & $68.2(10.5)$ & 14.9 & $<0.001$ & $69.3(10.2)$ & $69.2(10.3)$ & 0.8 & 0.85 \\
\hline \multicolumn{9}{|l|}{ Age group (years) } \\
\hline $45-54$ & $8.8(318)$ & $13.1(195)$ & 13.8 & $<0.001$ & 9.9 & 10.5 & 1.8 & 0.97 \\
\hline $55-64$ & $22.3(806)$ & $23.2(345)$ & 2.1 & & 22.5 & 22.5 & 0.0 & \\
\hline $65-74$ & $36.4(1318)$ & $33.7(501)$ & 5.8 & & 35.9 & 34.8 & 2.3 & \\
\hline $75-84$ & $27.8(1005)$ & $26.4(393)$ & 3.1 & & 27.3 & 27.4 & 0.4 & \\
\hline $85+$ & 4.7 (169) & $3.6(54)$ & 5.2 & & 4.4 & 4.8 & 1.7 & \\
\hline Sex (female) & $37.5(1355)$ & $35.3(526)$ & 4.4 & 0.15 & 36.6 & 35.8 & 1.5 & 0.71 \\
\hline \multicolumn{9}{|l|}{ Past history } \\
\hline Coronary artery disease & $39.2(1419)$ & $44.1(656)$ & 9.8 & $<0.001$ & 39.9 & 42.6 & 5.4 & 0.18 \\
\hline Peripheral vascular disease & $38.2(1383)$ & $35.7(531)$ & 5.3 & 0.09 & 38.2 & 38.2 & 0.1 & 0.99 \\
\hline $\begin{array}{r}\text { Stroke thought due to atherothrombotic } \\
\text { disease }\end{array}$ & $14.9(540)$ & $16.1(240)$ & 3.3 & 0.28 & 15.3 & 15.6 & 0.8 & 0.83 \\
\hline Transient ischemic attack history & $11.5(415)$ & $11.8(175)$ & 0.9 & 0.77 & 11.8 & 12.9 & 3.4 & 0.39 \\
\hline Hospitalized for congestive heart failure & $2.4(86)$ & $2.8(41)$ & 2.4 & 0.43 & 2.5 & 2.8 & 2.3 & 0.56 \\
\hline Documented heart failure & $5.6(204)$ & $4.4(66)$ & 5.5 & 0.08 & 5.3 & 5.4 & 0.3 & 0.95 \\
\hline Preoperative serum creatinine $>175 \mu \mathrm{mol} / \mathrm{L}$ & $4.5(162)$ & $5.2(78)$ & 3.5 & 0.248 & 4.5 & 3.4 & 5.7 & 0.06 \\
\hline $\begin{array}{r}\text { Diabetes on oral hypoglycaemic agent or } \\
\text { insulin }\end{array}$ & $27.5(993)$ & $26.9(401)$ & 1.2 & 0.71 & 27.4 & 26.5 & 2.0 & 0.63 \\
\hline Hypertension & $64.1(2317)$ & $61.5(915)$ & 5.3 & 0.08 & 63.4 & 65.5 & 4.5 & 0.24 \\
\hline \multicolumn{9}{|l|}{ Smoking Status } \\
\hline Never & $36.0(1301)$ & $37.4(556)$ & 2.9 & 0.42 & 35.9 & 37.1 & 2.4 & 0.49 \\
\hline
\end{tabular}




\begin{tabular}{|c|c|c|c|c|c|c|c|c|}
\hline Current & $21.3(770)$ & $19.8(294)$ & 3.8 & & 20.9 & 22.0 & 2.7 & \\
\hline Former & $42.7(1545)$ & $42.9(638)$ & 0.3 & & 43.2 & 40.9 & 4.6 & \\
\hline \multicolumn{9}{|l|}{ Preoperative medications } \\
\hline Aspirin & $35.7(1291)$ & $33.5(499)$ & 4.6 & 0.14 & 35.8 & 34.4 & 3.0 & 0.45 \\
\hline Clopidogrel & $3.9(141)$ & $5.2(77)$ & 6.1 & 0.04 & 4.4 & 4.7 & 1.6 & 0.71 \\
\hline LMWH/unfractionated heparin & $7.7(277)$ & $5.3(79)$ & 9.6 & $<0.001$ & 7.4 & 9.9 & 8.9 & 0.10 \\
\hline ACEI/ARB & $46.7(1689)$ & $40.4(601)$ & 12.8 & $<0.001$ & 44.7 & 45.1 & 0.8 & 0.84 \\
\hline Statin & $35.2(1274)$ & $33.3(496)$ & 4.0 & 0.20 & 34.8 & 33.5 & 2.7 & 0.48 \\
\hline Diuretic & $21.7(783)$ & $19.2(285)$ & 6.2 & 0.05 & 20.8 & 22.7 & 4.7 & 0.27 \\
\hline Calcium channel blocker & $20.1(726)$ & $24.5(365)$ & 10.7 & $<0.001$ & 21.4 & 21.0 & 0.78 & 0.83 \\
\hline Long-acting nitrates & $5.8(210)$ & $10.3(153)$ & 16.5 & $<0.001$ & 7.0 & 6.4 & 2.3 & 0.51 \\
\hline Non-study beta-blockers & $0.6(20)$ & $0.5(8)$ & 0.2 & 0.95 & 0.5 & 0.5 & 0.9 & 0.76 \\
\hline Digoxin & $2.7(99)$ & $2.5(37)$ & 2.5 & 0.61 & 2.6 & 2.7 & 0.2 & 0.97 \\
\hline Amiodarone & $1.4(49)$ & $1.1(16)$ & 1.5 & 0.42 & 1.3 & 1.6 & 3.0 & 0.57 \\
\hline Oral anticoagulants & $3.2(115)$ & $2.8(41)$ & 2.5 & 0.42 & 3.0 & 2.9 & 1.0 & 0.76 \\
\hline \multicolumn{9}{|l|}{ Type of surgery } \\
\hline Major vascular & $39.2(1419)$ & $38.4(572)$ & 1.6 & $<0.001$ & 39.9 & 38.7 & 2.5 & 0.68 \\
\hline Other vascular & $7.3(264)$ & $5.0(75)$ & 9.4 & & 6.6 & 8.2 & 5.9 & \\
\hline Orthopedic & $12.5(451)$ & $12.8(191)$ & 1.1 & & 12.7 & 11.7 & 3.1 & \\
\hline Intra-abdominal & $28.3(1025)$ & $24.8(369)$ & 8.0 & & 26.7 & 27.8 & 2.3 & \\
\hline Other & $12.6(457)$ & $18.9(281)$ & 17.2 & & 14.0 & 13.6 & 1.0 & \\
\hline \multicolumn{9}{|l|}{ Region } \\
\hline Australia and New Zealand & $13.2(479)$ & $15.0(223)$ & 5.0 & $<0.001$ & 13.7 & 13.4 & 1.0 & 0.98 \\
\hline Central and South America & $19.7(711)$ & $2.6(39)$ & 56.3 & & 14.7 & 16.7 & 5.5 & \\
\hline Canada & $51.2(1852)$ & $43.3(644)$ & 15.9 & & 48.9 & 47.7 & 2.4 & \\
\hline Southeast Asia, China and Hong Kong & $9.0(325)$ & $18.1(269)$ & 26.8 & & 11.7 & 11.3 & 1.1 & \\
\hline Europe & $5.1(185)$ & $4.0(60)$ & 5.2 & & 4.8 & 4.8 & 0.2 & \\
\hline India & $1.8(64)$ & $17.0(253)$ & 54.1 & & 6.2 & 6.1 & 0.4 & \\
\hline Emergency surgery & $10.5(380)$ & $8.3(124)$ & 7.5 & 0.02 & 9.9 & 10.1 & 0.6 & 0.90 \\
\hline
\end{tabular}




\begin{tabular}{|c|c|c|c|c|c|c|c|c|}
\hline Preoperative heart rate (beats per minute) & $77.1(12.5)$ & $78.4(12.7)$ & 10.3 & $<0.001$ & $78(12.6)$ & $78(12.1)$ & 0.8 & 0.82 \\
\hline Preoperative systolic blood pressure ( $\mathrm{mm} \mathrm{Hg}$ ) & $138.6(19.9)$ & $139.6(20.0)$ & 5.4 & 0.08 & 139 (19.9) & $139(20.4)$ & 1.7 & 0.69 \\
\hline
\end{tabular}

*Except for age, heart rate and systolic blood pressure which are presented as mean (standard deviation). LMWH = low molecular weight heparin. Std Diff = standardized difference. $\mathrm{ACEI}=$ angiotensin converting enzyme inhibitor. $\mathrm{ARB}=$ angiotensin-II receptor blocker. ${ }^{* *}$ Chi-squared test (categorical variables)

or ANOVA (continuous variables), unweighted or weighted as appropriate. 
Table 2 Estimated associations with outcome for nitrous oxide

\begin{tabular}{|c|c|c|c|c|c|c|c|c|}
\hline & \multicolumn{2}{|c|}{$\begin{array}{c}\text { No nitrous oxide } \\
(\mathrm{N}=3,616)\end{array}$} & \multicolumn{2}{|c|}{$\begin{array}{l}\text { Nitrous oxide } \\
(\mathrm{N}=1,488)\end{array}$} & \multicolumn{2}{|l|}{ Unadjusted } & \multicolumn{2}{|c|}{ Propensity score adjusted } \\
\hline & $\mathrm{N}$ & $\%$ & $\mathrm{~N}$ & $\%$ & $\mathrm{OR}(\mathrm{Cl})$ & $P$ value* & $\mathrm{OR}(\mathrm{Cl})$ & $P$ value* \\
\hline Primary** & & & & & (99\%: 0.82,1.50) & & (99\%: 0.75,1.57) & Pre \\
\hline \multirow[t]{2}{*}{ Myocardial infarction } & 204 & 5.6 & 89 & 6.0 & 1.06 (95\%: $0.82,1.38)$ & 0.636 & 0.99 (95\%: $0.75,1.31)$ & 0.94 \\
\hline & & & & & (99\%: 0.76,1.49) & 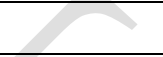 & (99\%: 0.69,1.42) & \\
\hline Stroke & & & & & (99\%: 0.16,1.66) & 8 & (99\%: 0.17,4.11) & \\
\hline \multirow[t]{2}{*}{ Death } & 100 & 2.8 & 40 & 2.7 & 0.97 (95\%: 0.67,1.41) & 0.878 & 1.04 (95\%: 0.6,1.81) & 0.88 \\
\hline & & & & & (99\%: 0.60,1.58) & & (99\%: 0.51,2.15) & \\
\hline \multirow[t]{2}{*}{ Hypotension } & 544 & 15.0 & 219 & 14.7 & 0.98 (95\%: $0.82,1.16)$ & 0.780 & 0.92 (95\%: $0.74,1.15)$ & 0.48 \\
\hline & & & & & (99\%: 0.78,1.22) & & (99\%: 0.70,1.23) & \\
\hline
\end{tabular}

OR = odds ratio; $\mathrm{Cl}=$ confidence interval; *Wald-test $\mathrm{p}$-value from unweighted/weighted logistic regression; $* *$ primary outcome $=$ cardiovascular death, non-fatal myocardial infarction and non-fatal cardiac arrest 
Table 3 Estimated associations with the outcome primary for nitrous oxide by region

\begin{tabular}{|l|c|c|c|r|}
\hline & No nitrous oxide & Nitrous oxide & \multicolumn{2}{|c|}{ Propensity score adjusted } \\
\hline Region & $\mathrm{N}(\%)$ & $\mathrm{N}(\%)$ & OR $(95 \% \mathrm{Cl})$ & $\mathrm{P}$ value \\
\hline Australia/New Zealand & $479(68.2)$ & $223(31.8)$ & $0.74(0.38,1.46)$ & 0.39 \\
\hline Central/South America & $711(94.8)$ & $39(5.2)$ & $0.80(0.18,3.54)$ & 0.77 \\
\hline Canada & $1,852(74.2)$ & $644(25.8)$ & $1.18(0.85,1.64)$ & 0.33 \\
\hline South east Asia/China/Hong Kong & $325(54.7)$ & $269(45.3)$ & $1.03(0.51,2.08)$ & 0.93 \\
\hline Europe & $185(75.5)$ & $60(24.5)$ & $2.20(0.82,5.92)$ & 0.12 \\
\hline India & $64(20.2)$ & $253(79.8)$ & $1.20(0.33,4.35)$ & 0.78 \\
\hline Overall & $3,616(70.9)$ & $1,488(29.2)$ & $1.08(0.82,1.44)$ & 0.58 \\
\hline P interaction & & & & 0.61 \\
\hline
\end{tabular}

$\mathrm{OR}=$ odds ratio; $\mathrm{Cl}$ = confidence interval; Primary outcome = cardiovascular death, non-fatal myocardial infarction and non-fatal cardiac arrest 